\title{
Quaternionic Wavelets for Texture Classification
}

\author{
Raphaël Soulard, Philippe Carré \\ XLIM-SIC laboratory - CNRS UMR 6172 - University of Poitiers, France
}

\section{Abstract}

This article proposes a study of the recent quaternionic wavelet transform (QWT) from a practical point of view through a digital image analysis application. Based on a theoretic 2D generalization of the analytic signal leading to a strong $2 \mathrm{D}$ signal modeling, this representation uses actual $2 \mathrm{D}$ analytic wavelets and yields subbands having a shift-invariant magnitude and a 3angle phase, using the quaternion algebra.

Our experiment furthers the understanding of this quite sophisticated tool, and shows its practical interest by a clear improvement of a famous wavelet application : texture classification. Thanks to coherent multiscale analysis brought by the QWT we obtain better classification results than with standard wavelets in a similar process.

Keywords: Wavelet transform, 2D Phase, Quaternionic Wavelet Transform, Image texture analysis, Image classification

Email addresses: raphael.soulard@univ-poitiers.fr (Raphaël Soulard), carre@sic.univ-poitiers.fr (Philippe Carré) 


\section{Introduction}

Since the 90's wavelets have been widely used for the analysis of textural images [Tuceyran and Jain, 1993; Lasmar and Berthoumieu, 2010]. Accordingly the human visual system sees textures through different channels related to particular frequency bands and directions; and wavelet representations offer this kind of decomposition. So by extracting simple features from each subband, the image content can be characterized separately at different scales and orientations, making an efficient texture analysis.

In 2001, the well known power of the analytic signal for signal modeling led to a new definition of wavelets : the Complex Wavelet Transform (CWT) [Selesnick et al., 2005], whose subbands are analytic signals giving access to a shift-invariant amplitude envelope (magnitude). The $\mathbb{C W T}$ codes signals in a more coherent way than standard wavelets (DWT); which overcomes their famous shift-variance problem. Its superiority over DWT for texture analysis has been shown in [de Rivaz and Kingsbury, 1999; Celik and Tjahjadi, 2009]. But $\mathbb{C W T}$ phase is ambiguous for $2 \mathrm{D}$ signals and is almost not used in applications excepted in a very recent work [Celik and Tjahjadi, 2011]. In parallel, the work in [Bülow, 1999] provides a strong theoretic 2D generalization of the analytic signal defined in quaternion algebra. The 3-angle local $2 D$ phase related to it contains 2D geometric information. The Quaternionic Wavelet Transform (QWT) - first proposed in 2004 [Chan et al., 2008;

Bayro-Corrochano, 2006] - uses wavelets that are 2D-analytic in the sense of Bülow, providing quaternion valued subbands with shift-invariant magnitude and this new 2D phase. This representation - specially defined for 2D signals - is a great theoretic improvement of $\mathbb{C W T}$ yielding a coherent description 
of local 2D structures.

Currently, many evolutions of wavelets focus on directionality (Contourlets, Complex Wavelets, Directionlets, Adaptive lifting schemes); bringing some redundancy to fit numerous directions from a quite algorithmic point of view. QWT is a different approach to a coherent representation of local structures in images based on a strong 2D signal processing theory; and sets some redundancy in a local phase rather than in directionality.

Given the promising theoretical properties of this new transform, we propose here a global study of this sophisticated tool to further its understanding through a famous application of wavelets. We aim to show its potential and verify its practical superiority over standard DWT in a comparative texture analysis application.

The main point is the understanding of the information carried by QWT coefficients. QWT magnitude can intuitively be used like DWT but interpretation of phase is far from straightforward. With a first QWT based feature extraction algorithm this work gives an application not did yet to our knowledge and furthers practical use of QWT coefficients. We expect QWT to provide better texture analysis than DWT - with simple feature extraction methods - thanks to better separation of information and shift-invariance. An additional experimental comparison is done with $\mathbb{C W T}$ because the topic is near.

After a presentation of the transform, we propose several feature extraction methods based on both DWT and QWT. To compare with literature, we also describe some $\mathbb{C W T}$ based feature descriptors recently published in [Celik and Tjahjadi, 2009, 2011]. The use of QWT phase is then discussed as 


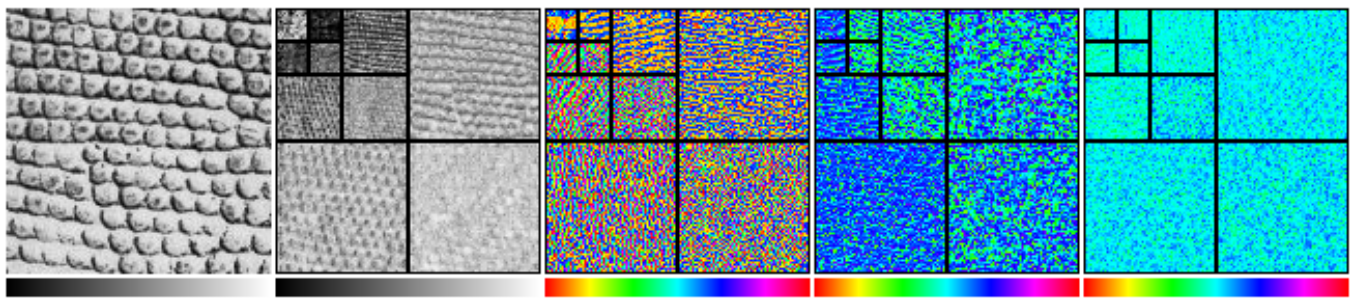

Figure 1: Quaternionic wavelet transform of a textural image. From left to right : Original image, magnitude $|q|$ (intensity inverted for visual convenience), $\varphi \in[-\pi ; \pi], \theta \in\left[-\frac{\pi}{2} ; \frac{\pi}{2}\right]$, $\psi \in\left[-\frac{\pi}{4} ; \frac{\pi}{4}\right]$. The 3 terms of phase are represented in color - the hue corresponding to the angle (cyan for 0 , red for $\pm \pi$ ). Darker zones in phase correspond to negligible magnitude (making phase absurd).

well as the combination of QWT magnitude and phase based features. We finally give experimental classification results to compare DWT and QWT in terms of recognition rates. Note that the point is to observe changes in recognition and not to get a competitive texture recognition performance. The aim is to carry out a simple process to help us studying wavelet transforms, rather than achieving excellent recognition. Types of texture better described by one or another transform will be analyzed in terms of visual/geometric content.

\section{Quaternionic Wavelet Transform}

The Quaternionic Wavelet Transform (QWT) is a perfect reconstruction 2D filterbank decomposition for grayscale images; addressing three common drawbacks of standard DWT :

- Oscillations - Using oscillating wavelets complicates the representation of a simple structure, involving several coefficients in a neighborhood 
to compensate each other their own oscillations, and so making local analysis difficult.

- Shift-variance - The look of a wavelet transform may substantially change by a small shift of the image; causing incoherence while most recognition processes need invariant features.

- No phase notion - Fourier phase and local phase from the analytic signal are powerful analysis tools so it is interesting to fit DWT up with a phase notion in a pattern recognition context.

The key is to embed the analytic signal modeling into the wavelet framework. The Dual-Tree $\mathbb{C W T}$ [Selesnick et al., 2005] achieves 1D analytic wavelet analysis with a 2 times redundant perfect reconstruction filterbank overcoming the 3 drawbacks listed above. But its 2D version yields an ambiguous phase failing to efficiently describe local structures. More precisely, the phase angle encodes a local shift around pixel position - in the exact direction that is orthogonal to the intrinsic orientation of considered subband. Since the structure may be not well aligned with wavelet orientation, this shift information is not sufficient to accurately localize the structure.

The point then is the 2D generalization of analytic signal and the geometric meaning of the $2 \mathrm{D}$ phase extracted from it.

This is why the QWT uses the 2D analytic signal defined in [Bülow, 1999] within the quaternion algebra. According to Bülow, this generalization is superior to previous attempts in $\mathbb{C}$ - including the one implicitly related to 2D $\mathbb{C W T}$ - and is well adapted for 2D signals whereas standard complex analytic signal is only adapted in 1D. The extracted quaternionic local phase 
(3 angles) contains rich geometric information. Note that Bülow proposed quaternionic Gabor filters in 1999 [Bülow, 1999] and used the phase for image analysis applications.

The QWT provides a quaternionic multiresolution analysis with 2D analytic wavelets overcoming the above-cited drawbacks of DWT. Whereas DWT coefficients are real, QWT is quaternion valued i.e. 4-vectors made of one magnitude and a 3-angle phase. Information is better separated to describe more explicitly the image content. In 2004, the Rice University from Houston proposes to use their Dual-Tree algorithm to carry out a QWT with perfect reconstruction filterbanks [Chan et al., 2008] (that we use in this work). Note that at the same time, Bayro proposes a quaternionic Gabor pyramid [BayroCorrochano, 2006], windowing the quaternionic Fourier transform (QFT), but this approach has no perfect reconstruction so the use can be limited for example in image coding.

In this section we present the quaternionic 2D analytic signal upon which the QWT is based, the definition of the transform, the algorithm we used and the use of this tool.

\subsection{The Quaternionic Analytic Signal}

In his thesis [Bülow, 1999], T. Bülow showed that complex algebra $\mathbb{C}$ is only adapted for handling $1 \mathrm{D}$ signals and that $2 \mathrm{D}$ signals are best described by embedding signal processing tools in the more general quaternion algebra HI. He defined a 'Quaternionic 2D Fourier Transform' (QFT) and a 'Quaternionic 2D Analytic Signal'.

A quaternion is a generalization of a complex number, related to 3 imaginary units $i, j, k$ following the rules $i^{2}=j^{2}=k^{2}=-1$ and $i j=-j i=k$; and 
written $q=a+b i+c j+d k$. A polar form $q=|q| e^{i \varphi} e^{j \theta} e^{k \psi}$ analogous to the complex one is defined by one modulus $|q|$ and three angles $(\varphi, \theta, \psi)$ called phase. The phase corresponds here to Euler angles in a 4D Euclidean space :

$$
\begin{array}{lll}
\varphi=\operatorname{atan}\left(2(c d+a b), a^{2}-b^{2}+c^{2}-d^{2}\right) / 2+k \pi & \in[-\pi ; \pi] \\
\theta=\operatorname{atan}\left(2(b d+a c), a^{2}+b^{2}-c^{2}-d^{2}\right) / 2 & \in\left[-\frac{\pi}{2} ; \frac{\pi}{2}\right] \\
\psi=\arcsin (2(a d-b c)) / 2 & \in\left[-\frac{\pi}{4} ; \frac{\pi}{4}\right]
\end{array}
$$

112 with $|q|=1$. See [Bülow, 1999] pp 16-21 for a detailed definition.

As a fundamental signal processing tool the analytic signal associated to a real 1D signal $f(t)$ is well known to be constructed by setting its Hilbert transform $\mathcal{H} f(t)$ in the imaginary part; being equivalent to a simple spectral operation :

$$
f_{A}(t)=f(t)+i \mathcal{H} f(t) \Leftrightarrow \hat{f}_{A}(\omega)= \begin{cases}0 & \text { if } \omega<0 \\ \hat{f}(\omega) & \text { if } \omega=0 \\ 2 \hat{f}(\omega) & \text { if } \omega>0\end{cases}
$$

Note that the spectrum of $f_{A}$ is null for negative frequencies.

Modulus and argument of $f_{A}$ can be interpreted as instantaneous magnitude and phase. When the original signal is considered oscillating or narrowband these two pieces of data become meaningful - making this tool useful for instance in amplitude and frequency modulation.

Looking at one point of interest, we can interpret a high magnitude as a strong 'presence of some oscillation' around this point. Phase indicates the relative location of this point within the oscillation.

Bülow pointed out that instantaneous phase equivalently describes the kind of structure at a point - among four fundamental structures : 0-'positive 
impulse', $\pi / 2$-'falling step', $\pm \pi$-'negative impulse', $-\pi / 2$-'rising step'. This is an important point for pattern recognition and that is why the author wanted to generalize it for $2 \mathrm{D}$ signals.

Several generalizations to 2D are possible and depend on properties we choose to keep. We consider 2D versions of the Hilbert transform (HT). The partial $\mathrm{HT}\left(\mathcal{H}_{\theta}\right)$ is equivalent to a $1 \mathrm{D}$ HT along direction $\theta$. The total HT $\left(\mathcal{H}_{T}=\mathcal{H}_{0} \mathcal{H}_{\pi / 2}\right)$ is the combination of two partial HT's along $x$ and $y$ axes. A $2 \mathrm{D}$ analytic signal of $f$ can be constructed by adding $\mathcal{H}_{\theta} f$ as the imaginary part; what would cancel out a $\theta$-oriented half-plane in the frequency domain. But this method depends on an arbitrary choice of the direction - not really adapted for our issue. We can use as well $\mathcal{H}_{T}$; what cancels out 3 quadrants of the Fourier domain, but the original signal cannot be retrieved from the analytic signal (This is the classical approach in [Hahn, 1996]).

So Bülow proposed to embed both partial and total HT's into a quaternionic analytic signal :

$$
f_{A}(x, y)=f(x, y)+i \mathcal{H}_{0} f(x, y)+j \mathcal{H}_{\pi / 2} f(x, y)+k \mathcal{H}_{T} f(x, y)
$$

This method cancels out 3 quadrants of the (quaternionic) Fourier domain and allows retrieval of the original signal. Its polar representation provides $2 \mathrm{D}$ local magnitude and phase that can be used to analyze $2 \mathrm{D}$ signals - in a similar way as with $1 \mathrm{D}$ analytic signal.

The QWT uses this generalization to carry out an 'actual' 2D analytic wavelet transform. 


\subsection{Quaternionic Wavelets : Definition}

A standard wavelet transform (DWT) provides a scale-space analysis of an image; yielding a matrix in which each coefficient is related to a 'subband' (localization in the 2D Fourier domain) and to a position in the image. A 'subband' means both an oscillation scale (i.e. a 1D frequency band) and a spatial orientation (i.e. rather vertical, horizontal or diagonal).

For 2D signals, the QWT provides a richer scale-space analysis than DWT. Its coefficients are analytic according to Bülow's theory and contrary to DWT the magnitude is near shift-invariant. Thus it inherits the magnitude-phase local analysis from the very useful 1D analytic signal. In the one hand the QWT can be viewed like a local '2D Quaternionic Fourier Transform' (QFT); in the other hand its subbands are '2D Quaternionic Analytic Signals' associated with bandpass filtered versions of the original signal.

Note that usual interpretation of magnitude remains analogous to 1D as it indicates the relative 'presence' of a local geometric element; whereas the local phase is now represented by 3 angles carrying a complete description of this $2 \mathrm{D}$ structure.

We start with real separable scaling function $\phi$ and mother wavelets $\psi^{D}$, $\psi^{V}, \psi^{H}$, and we construct their analytic extensions in $\mathbb{H}$ :

$$
\begin{array}{ll}
\text { Real function : } & \text { Analytic extension : } \\
\psi^{D}=\psi_{h}(x) \psi_{h}(y) \rightarrow \psi^{D}+i \mathcal{H}_{0} \psi^{D}+j \mathcal{H}_{\pi / 2} \psi^{D}+k \mathcal{H}_{T} \psi^{D} \\
\psi^{V}=\phi_{h}(x) \psi_{h}(y) \rightarrow \psi^{V}+i \mathcal{H}_{0} \psi^{V}+j \mathcal{H}_{\pi / 2} \psi^{V}+k \mathcal{H}_{T} \psi^{V} \\
\psi^{H}=\psi_{h}(x) \phi_{h}(y) \rightarrow \psi^{H}+i \mathcal{H}_{0} \psi^{H}+j \mathcal{H}_{\pi / 2} \psi^{H}+k \mathcal{H}_{T} \psi^{H} \\
\phi=\phi_{h}(x) \phi_{h}(y) \rightarrow \phi+i \mathcal{H}_{0} \phi+j \mathcal{H}_{\pi / 2} \phi+k \mathcal{H}_{T} \phi
\end{array}
$$


Mathematically, 2D HT's of separable functions are equivalent to 1D HT's along rows and/or columns. So we consider the 1D Hilbert pair of wavelets and scaling functions :

$$
\left(\psi_{h}, \psi_{g}=\mathcal{H} \psi_{h}\right) \quad\left(\phi_{h}, \phi_{g}=\mathcal{H} \phi_{h}\right)
$$

and we have for instance : $\mathcal{H}_{0} \psi^{V}=\mathcal{H} \phi_{h}(x) \psi_{h}(y)=\phi_{g}(x) \psi_{h}(y)$. The analytic $2 \mathrm{D}$ wavelets can then be written in terms of separable products :

$$
\begin{aligned}
\psi^{D} & =\psi_{h}(x) \psi_{h}(y)+i \psi_{g}(x) \psi_{h}(y)+j \psi_{h}(x) \psi_{g}(y)+k \psi_{g}(x) \psi_{g}(y) \\
\psi^{V} & =\phi_{h}(x) \psi_{h}(y)+i \phi_{g}(x) \psi_{h}(y)+j \phi_{h}(x) \psi_{g}(y)+k \phi_{g}(x) \psi_{g}(y) \\
\psi^{H} & =\psi_{h}(x) \phi_{h}(y)+i \psi_{g}(x) \phi_{h}(y)+j \psi_{h}(x) \phi_{g}(y)+k \psi_{g}(x) \phi_{g}(y) \\
\phi & =\phi_{h}(x) \phi_{h}(y)+i \phi_{g}(x) \phi_{h}(y)+j \phi_{h}(x) \phi_{g}(y)+k \phi_{g}(x) \phi_{g}(y)
\end{aligned}
$$

From a practical point of view, the mother wavelet is related to a quaternionic 2D analytic filter - computable with separable 2D filterbanks. This means the decomposition is heavily dependent on the position of the image with respect to $x$ and $y$ axes (rotation-variance) and the wavelet is not isotropic but the advantage is the easy computation.

Each subband from QWT can be seen as the analytic signal associated with a narrowband part of the image. Shift-invariant magnitude $|q|$ represents elements at any space position in each frequency subband; and phase $(\varphi, \theta, \psi)$ describes the 'structure' of these elements. We discuss below the interpretation of the phase.

\subsection{Dual-Tree Implementation}

The QWT is implemented by the Dual-Tree algorithm [Selesnick et al., 2005] - a filterbank using a Hilbert pair as a complex 1D wavelet - originally 


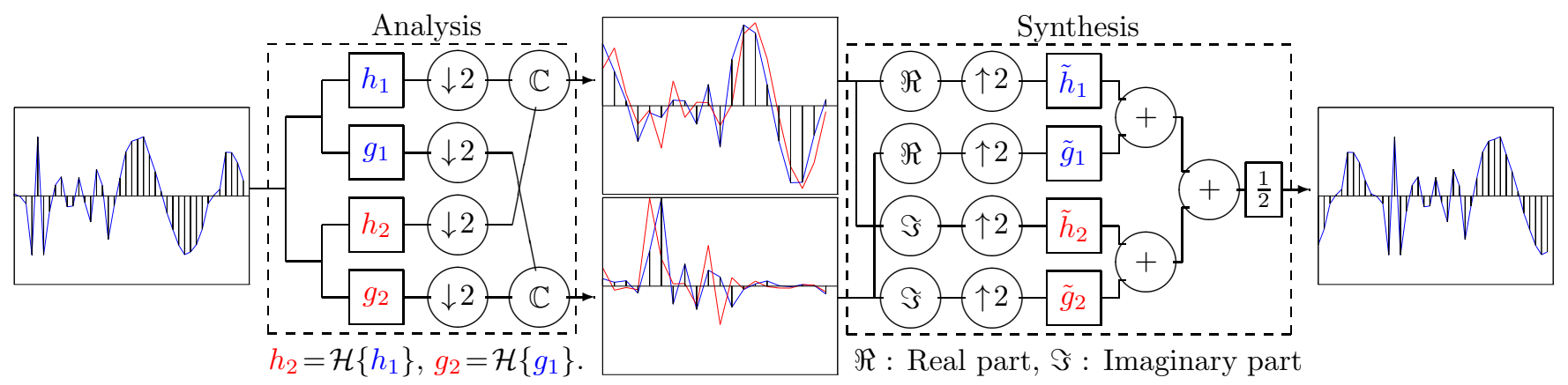

Figure 2: 1D analytic wavelet transform with the Dual-Tree algorithm.

performing a $\mathbb{C W T}$ as shown Fig. 2 for the 1D case. It is approximately analytic and achieves near shift-invariance with a redundancy of 2:1. These two filterbanks lead to four separable 2D filterbanks that can be combined to fit the QWT definition. Fig. 1 shows an example of such decomposition.

\subsection{Interpretation and Use}

For now, literature is quite poor about QWT and the major difficulty with the use of this transform is the interpretation of the phase.

In [Bülow, 1999] 2D quaternionic analytic Gabor filters are defined and used in a Gabor based texture segmentation. Filtered images are 2D analytic and form a scale-space analysis of the image; from which are extracted magnitudes and local phases at each point to characterize the texture.

A shift theorem similar to this of the well known Fourier transform also stands for the quaternionic Fourier transform defined in [Bülow, 1999] : a shift of the image is equivalent to an offset of the two first terms of phase $\varphi$ and $\theta$. As a result, in a Gabor decomposition $\varphi$ and $\theta$ describe small 2D spatial shifts of the coded structure - around the quaternionic coefficient position. Note that in 1D that shift is equivalent to structural information; 
but 2D structures may be more complex than lines or edges (e.g. corners, T-junctions) and cannot be described by this kind of shift coding. The third term $\psi$ completes the structure analysis and is seen as a texture feature. Bülow observed that where $\psi$ is around $\pm \pi / 4$ the coded structure is a line or an edge oriented along a diagonal.

We found three recent references [Chan et al., 2008; Bayro-Corrochano, 2006; Zhou et al., 2007] where $\varphi$ and $\theta$ are used in disparity estimation. As the QWT performs local QFT's; the shift theorem approximately stands for QWT so $\varphi$ and $\theta$ code quite simply spatial shifts of structures.

In another application of [Chan et al., 2008] ('wedgelet' representation) $\varphi$ and $\theta$ are used for wedges position and $\psi$ is used for their orientation.

We propose a new application of this transform : the next section deals with texture classification. The difference with the work by Bülow is that while segmentation involves detecting where two textures are different, classification aims to indicate what kind of texture is observed. In addition, the tool we use (QWT) is slightly different than Gabor filters in that it is related to perfect reconstruction filterbanks.

\section{Texture Classification}

Texture classification is the process which - given any textural image - find the class this image most probably belongs to. Texture has still no universal definition, but may be presented by classical cases like 'tar', 'water', 'sand', as macroscopic examples, or 'town','ocean', 'forest', as satellite view examples; and characterized by a sort of uniformity and periodicity. We call a kind of texture a class; according to an arbitrary classification we humans 
make instinctively.

In this paper, we only discuss wavelet based methods for this application in order to compare QWT with DWT. Feature extraction via standard wavelet representation of images (DWT) has been widely used in texture analysis [Tuceyran and Jain, 1993; Arivazhagan and Ganesan, 2003; Lasmar and Berthoumieu, 2010] for the two last decades. Accordingly, the multiscale analysis provided by the DWT is well adapted to textural images. From each subband one may calculate a mean, standard deviation, energy, mean power, or more sophisticated statistical modeling. Those features - well combined can form a powerful texture descriptor.

The Dual-Tree Complex Wavelet Transform (CWT) - a complex extension of the DWT - is motivating by the near shift-invariance of its magnitude and the oriented aspect of its wavelets. It was used in [de Rivaz and Kingsbury, 1999; Celik and Tjahjadi, 2009, 2011] revealing its efficiency with respect to DWT in texture analysis. The invariance of magnitude to shifts makes the extracted feature independent of the precise location of textural patterns; and so allows a better characterization. We will include $\mathbb{C W T}$ based feature vectors of [Celik and Tjahjadi, 2009, 2011] in our experiments to compare with literature.

We here propose to further texture analysis using QWT because we keep the advantages due to shift-invariance while adding new information with the $\psi$ phase. The QWT can be regarded as the proper $2 \mathrm{D}$ generalization of analytic wavelets since the quaternionic analytic signal is the proper generalization according to Bülow. We use the Dual-Tree based QWT algorithm [Chan et al., 2008] and for comparison a DWT with the well known CDF 9/7 
wavelets. The latter are widely used and offer a good separation of frequency components; what is well adapted to texture analysis.

QWT needs special "Q-Shift" filters designed in [Kingsbury, 2001] to form Hilbert pairs. Three different filter sets are possible : the 9-tap, 14-tap and 18-tap Q-Shift filters. In wavelet based texture analysis long filters usually achieve better performance. In this paper we mainly use the 9-tap filters to ensure fairer comparison with $9 / 7$ classical filters; nevertheless the other Q-Shift filters will be treated in the experimental part of the paper.

A $L$-level decomposition provides $3 L$ subbands for analysis and a lowfrequency subband. We do not use the latter; as low frequency structures almost constant - are usually not considered to be a feature of texture.

\subsection{Texture Databases}

In order to provide relevant performance with respect to the huge variety of textures we can find, we use several known texture databases :

- Brodatz database [Brodatz, 1966] contains 111 photos called textures by the author. We use those $[640 \times 640]$ images to create texture classes, by cutting each one into 25 [128 × 128] sub-images considered to belong to the same class.

- Outex databases (http://www.outex.oulu.fi/) were specially made for texture classifiers benchmarking. We use the TC12 database that contains 24 classes of 380 [128 × 128] samples. In each TC12 class we have different images of a same kind of texture at different orientations and under different lightings. 
- We also use Outex TC14 database, which has 68 classes of 60 samples with different lightings but only one orientation.

\subsection{Experimental Protocol}

Recall that our point is the understanding of the QWT. The recognition scheme must be elementary so we can identify the influence of the choice of transform/features on the recognition rate. This is why we propose to use a simple $k$ Nearest Neighbors classifier $(k$-NN) without any automatic feature selection or normalization that would drown the contribution of the transform and confuse the interpretation. Note that we use DWT-based and QWT-based feature vectors that will be presented in the next section.

Here is the $k$-NN algorithm. From each image of a training base a feature vector describing the texture is extracted and labelled by its actual class number. When a test image is given, its feature vector is processed and compared in the feature space to the feature vectors of the training base in terms of Euclidean distance. According to a parameter $k$, the $k$ nearest vectors are kept to find the most representative class; that is the most represented class within the $k$ neighbors.

In order to evaluate performance we split our available classes into two groups :

- A test base : $N_{T}$ examples the program does not know;

- A training base : $N_{A}$ labelled examples the program knows.

Then recognition rates obtained by using a certain feature extraction method will inform about the quality of this method i.e. the power of description 
brought by this feature vector for texture classes. Some classes are better recognized than others so we can study what kinds of texture make DWT or QWT more suitable.

\section{Cross validation}

Since experimental rates will depend on the chosen training base, we propose to iterate the process 100 times with different selections of the training base (but fixed $N_{A}$ ). We obtain 100 recognition rates for each feature extraction method and each texture class. So our recognition rates will be displayed as average rates with a certain deviation, following the so-called cross validation protocol (see Fig. 3 for example). Thanks to cross validation recognition rates are very stables from an experiment to another (rates vary less than $1 \%$ experimentally).

\section{Sample selection}

Let us precise how the sample selection is done. We need to separate samples of each class 100 times differently. If we naïvely pick up $N_{A}$ consecutive samples as they are stored in the database, we introduce a bias due to the existing correlation between consecutive samples (e.g. in TC12 basis, consecutive samples have same orientation). So $N_{A}$ samples are randomly selected from each class while the test base is formed by all remaining samples. This process is repeated at each of the 100 iterations.

In the sequel, we first present different feature extraction methods from DWT/QWT magnitude and QWT phase, as well as complex wavelet features from [Celik and Tjahjadi, 2009, 2011] for comparison. Then the experimental classification results related to these descriptors are given and analyzed. 


\section{Feature extraction}

Feature extraction is the automatic processing of several numbers describing texture characteristics. The output is a so-called feature vector that will be considered in a feature space to compare with other texture samples. Many wavelet based texture classifications use a global measure of energy or statistics from the magnitude; which corresponds to characterize particular bands of the Fourier domain. This type of feature extraction is well adapted to textures that can be viewed as superimposed signals located in the Fourier domain. We will study several methods. First we use magnitude based features that are analogous for both DWT and QWT. Then we propose QWT phase based features that will improve texture description; and a combination of QWT magnitude and phase. Finally the $\mathbb{C W T}$-based features of [Celik and Tjahjadi, 2009, 2011] - also made of global subband measures - are described.

\subsection{Magnitude based features}

After having processed the norm $M_{i j}=\left|q_{i j}\right|$ of wavelet coefficients of a given image (where $i$ and $j$ are discrete coordinate of a pixel); two measures in each subband are considered.

\section{Energy :}

$$
m=\frac{1}{E} \sum_{i, j} M_{i j}^{2}
$$

where $E$ is the total energy of the image minus the low-frequency energy. This normalization makes $m$ the relative amount of energy in a subband. 
Standard deviation (St. dev.) :

$$
m=\sqrt{\frac{1}{N} \sum_{i, j}\left(M_{i j}-\mu\right)^{2}}
$$

where $N$ is the number of pixels in the subband and $\mu$ is the mean.

\subsection{QWT phase based features}

We dealt with QWT phase in section 2.4. We will first use some phase features only and then we combine magnitude and phase information. Now, how can we use QWT phase to describe textures?

First, $\varphi$ and $\theta$ are irrelevant because they inform about position of elements whereas we are interested in their structure. We may think of a matching between the 2D shift coding and some 2D kinds of structure - analogously to the 1D phase - but this interpretation is not clear. In addition, our experiments on $\varphi$ and $\theta$ gave very bad results (less than $3 \%$ recognition). So we focus on the third term $\psi$ used by Bülow in a Gabor based texture segmentation process [Bülow, 1999].

In this application we assume that textures are stationary so we do not want to describe spatially precise texture patterns but rather extract global measures. However, we want to describe a part of the behavior of the phase within each subband so a measure such as a mean would not be suitable because it is too global. Experimentally, phase means ${ }^{1}$ by subband give very bad results (less than $1 \%$ recognition).

\footnotetext{
${ }^{1}$ The angle is 'unwrapped' in the calculus of a mean. But note that $\psi$ does not show phase wrapping as it lies in $[-\pi / 4 ; \pi / 4]$.
} 
As we aim to do a fair comparison we won't carry out a high level process such as spatial measures (extrema search, connexity ...) that would need to be differently adapted to either DWT or QWT. To make the results as comparable as possible our application must keep simple and we would rather extract a single value from a whole subband as a global feature of the phase spatial behavior. To our knowledge, literature is quite poor about the measure of phase. The Global Phase Coherence [Blanchet et al., 2008] gives a measure of image sharpness but relies on a too much restricted mathematical model with respect to the topic of the paper that is a first approach toward QWT. A relative phase distribution measure was used with some complex wavelets in [Vo et al., 2007] but is specially defined for this type of transform. The quaternionic phase is a totally different concept than the complex one; so it would be irrelevant to use such a sophisticated measure.

It seems that the simple calculus of the standard deviation (st. dev.) within a subband would be adapted because it describes a part of the behavior of $\psi$. Since $\psi \in\left[-\frac{\pi}{4}, \frac{\pi}{4}\right]$ (see Euler angles) we avoid the usual problems about circular data ( $\pm \pi$ discontinuity, phase wrapping) so there is no ambiguity to calculate angle differences and means.

To improve robustness the $\psi$-deviation can be weighted by the QWT magnitude. A high magnitude means an important presence of an element while a low value means 'no element' and also provides a numerically unstable phase. So the measure should be more representative by not considering the structure of low magnitude features. The weight function $W$ is the magnitude of the QWT coefficients normalized so the sum within the subband is 1; and is integrated in the standard deviation formula as defined below. Here are 
the two phase measures we use for feature extraction :

St. dev. :

$$
m=\sqrt{\frac{1}{N} \sum_{i, j}\left(\psi_{i j}-\mu\right)^{2}}
$$

where $\mu=\frac{1}{N} \sum_{i, j} \psi_{i j}, N$ is the number of pixels in the subband, and $(i, j)$ spans a subband.

Weighted st. dev. :

$$
m=\sqrt{\sum_{i, j} W_{i j}\left(\psi_{i j}-\mu_{W}\right)^{2}}
$$

where $\mu_{W}=\sum_{i, j} W_{i j} \psi_{i j}$ is the weighted mean of the subband.

\subsection{Combination of $Q W T$ magnitude and phase}

Let us now combine the st. dev. QWT magnitude features with the weighted st. dev. QWT phase features (Given experimental results that will be presented in the next section; st. dev. measures are retained for both magnitude and phase).

First we experimentally observed that a simple concatenation of both vectors gives very good performance - better than DWT. Here is a classical point in classification : our two features are not of the same kind. One is an amplitude $\left(\in \mathbb{R}^{+}\right)$and the other is an angle $\left(\in\left[0, \frac{\pi}{2}\right]\right)$. It produces a lack of coherence in the final feature vector as all that terms are seen the same way by the Euclidean distance of the $k$-NN algorithm. A metric often solves this heterogeneity problem but the different metrics we tried did not improve the process. Even considering the optimal metric with respect to our database; performance is not significantly higher since in practice magnitude 
and phase features are of the same order of magnitude. So for this paper we finally consider simple concatenation of QWT magnitude and phase features.

To compare with literature we propose to also use $\mathbb{C W T}$. Even though the concept is totally different from QWT the underlying algorithm is quite similar and was used in texture analysis for example in [de Rivaz and Kingsbury, 1999; Celik and Tjahjadi, 2009, 2011].

\subsection{Complex wavelets based features}

These feature vectors are processed from the 6 subbands of the DualTree Complex Wavelet Transform (CWT). In most cases, classical features such as entropy or energy measures as well as statistics are extracted from the magnitude of each subband. In this experiment we choose to use $\mathbb{C W T}$ magnitude based feature vector from [Celik and Tjahjadi, 2009] that uses variance and entropy. The very recent work in [Celik and Tjahjadi, 2011] involves standard deviations and energy measures from both magnitude and phase. Note that in the original paper the vector is further reduced and weighted based on statistics (PCA) - which we did not reproduce because our scheme needs to be elementary so we can identify the contribution of DWT/CWT/QWT (see discussion section 3.2).

It is important to stress that the aim of cited papers is to carry out a good recognition whereas the point of the present paper is the analysis of the QWT. This is why we do not use the whole recognition process described in [Celik and Tjahjadi, 2009, 2011] but just the feature extraction bloc. We refer the reader to original publications for detailed expressions.

Now the feature extraction part is done, let us give our experimental results of texture recognition with every feature vectors described above. 


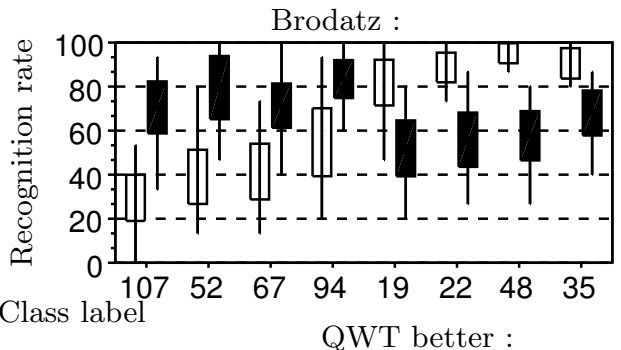

QWT better :

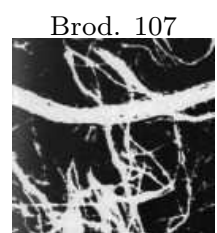

$\mathrm{TC} 1215$

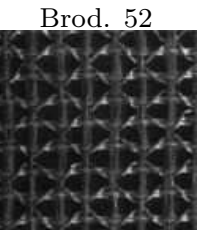

TC12 14

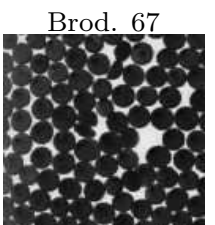

TC14 14

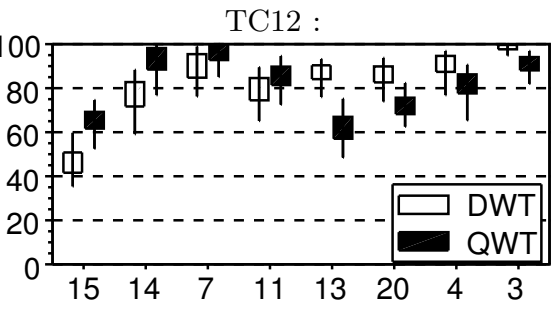

Brod. 94

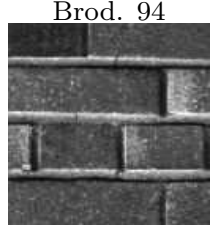

TC14 2
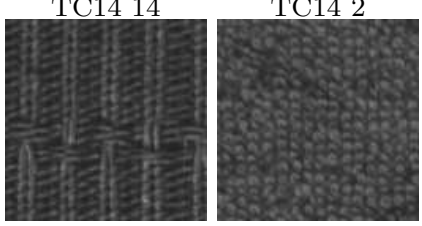

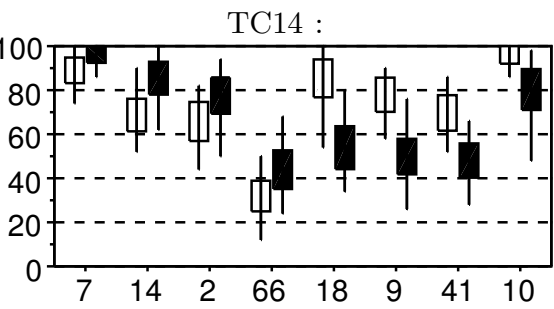

DWT better :
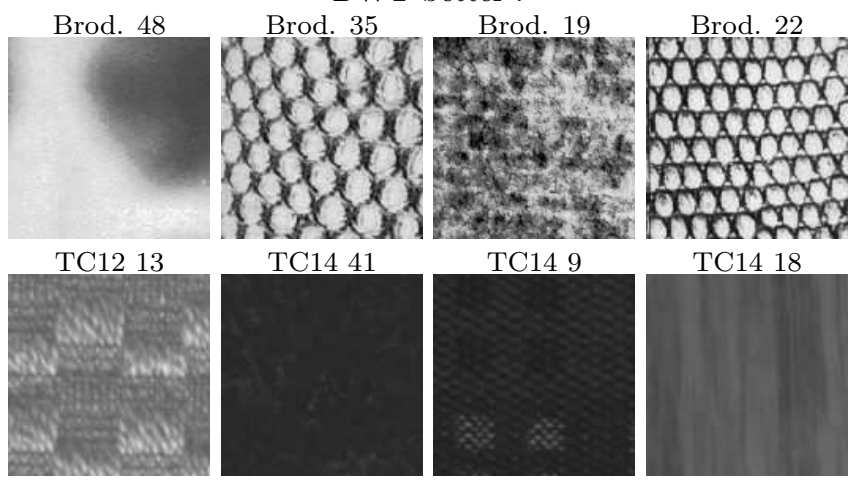

Figure 3: Magnitude based classification performance : cross validation results for some classes of the 3 databases.

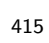

416

417

\section{Experimental results}

We obtained many results, depending on $L$ (depth of wavelet decomposition), chosen feature extraction, value of $k$, size of training base $N_{A}$ and used database.

Experimentally for most feature vectors best results are obtained with $L=3$ and $k=3$. The larger the training base the better the recognition, so in this comparative study we consider rather small training bases to put the simulation into a rather hard context. The st. dev. measure globally gives slightly better performance than energy, so we will only consider the st. dev. 
feature in the sequel.

The choice of Q-Shift filters in Dual-Tree algorithm has almost no influence on recognition rates (gain is less than $1 \%$ recognition). A reason is that this modification does not affect the first scale (see [Kingsbury, 2001]) so $\frac{1}{3}$ of features remain the same. So we keep 9-tap filters.

For every result presented in the paper, we keep :

- Depth is $L=3$ (9 subbands) so feature vectors are of size 9 for DWT/QWT magnitude and phase features, size 18 for QWT combined magnitude and phase, size 36 for $\mathbb{C W T}$ magnitude from [Celik and Tjahjadi, 2009] and size 72 for $\mathbb{C W T}$ magnitude and phase from [Celik and Tjahjadi, 2011].

- Number of considered neighbors is $k=3$;

- Size of the training base per class is $N_{A}=10$ (resp. $N_{A}=30$ and $\left.N_{A}=10\right)$ for Brodatz (resp. TC12 and TC14) database;

- Magnitude measure is a standard deviation;

- 9-tap Q-Shift filters are used in the Dual-Tree algorithm.

We now present experimental results in terms of recognition rates for DWT/QWT magnitude based features, QWT phase features, combination of QWT magnitude and phase and $\mathbb{C W T}$ based features.

\subsection{Magnitude based descriptor: comparing DWT and $Q W T$}

Some cross validation results for magnitude based feature extraction are illustrated Fig. 3. Recognition rates are expressed in terms of mean, standard 
deviation and extrema of the rates obtained for each class with the various training bases. Class labels are on the $x$-axis and recognition rate on the $y$-axis. For each class the vertical line spans all 100 obtained rates between the minimum and the maximum, the bar is centered on the average rate and is high two times the standard deviation.

Note that globally, both transforms have equivalent performance. We do not present results for all classes because they are too numerous but we selected the cases where difference of performance is maximum between DWT and QWT. These extreme cases could give clues about what type of texture is better recognized by either the DWT or the QWT.

We propose to analyze the results through particular cases to highlight QWT characteristics. Observing Fig. 3, we see that texture Brod. 67 contains numerous very similar and randomly placed small black disks. Shiftinvariance of QWT magnitude is essential to code those structures with various positions; whereas each disk is surely quite differently coded in the DWT because of small space shifts. In this case QWT gives a more robust description of texture - keeping everywhere a coherent representation of the disks what explains the superiority of QWT for Brod. 67.

Let us call 'common' orientations multiples of $45^{\circ}$ i.e. horizontal, vertical and diagonal. We can globally see that textures better recognized by QWT have many structures right aligned with 'common' orientations. Those for which QWT is less efficient rather contain structures oriented between them.

According to us, this is because QWT phase contains some information about these intermediate orientations. So by using only magnitude, superiority of QWT is not visible at those cases where many 'intermediate' structures 
are present. But for other cases, this superiority is experimentally confirmed as the structures are better encoded by QWT magnitude than by DWT giving higher recognition rates.

To conclude about magnitude based feature extraction, global recognition performance is quite similar between DWT and QWT. But if we look closer at particular textures we see that shift-invariance of QWT improves recognition of textures made of 'randomly located similar structures'; and that structures aligned with a multiple of $45^{\circ}$ are better represented in QWT magnitude while other orientations must have their related information in the phase. And works in [Bülow, 1999] and [Chan et al., 2008] show that QWT phase can provide a powerful image analysis; making clear that the QWT is not fully exploited here. Now let us use QWT phase to improve recognition and outperform DWT.

\subsection{Contribution of QWT-phase}

Quite surprisingly phase based features give performance comparable to magnitude as shown Fig. 4 - that also contains next results. This is a very good result. Weighted st. dev. gives much better description than simple st. dev. what confirms the relevance of less considering phase data of low magnitude coefficients. In the sequel we only consider weighted st. dev. as phase measure.

All cases occur : for a same texture both magnitude and phase analysis may be efficient or not - the results are very heterogeneous. It is interesting to note that many textures are very well analyzed either by QWT magnitude or by QWT phase - what suggests that the two feature vectors are complementary. The former gives frequency information like DWT and the latter 


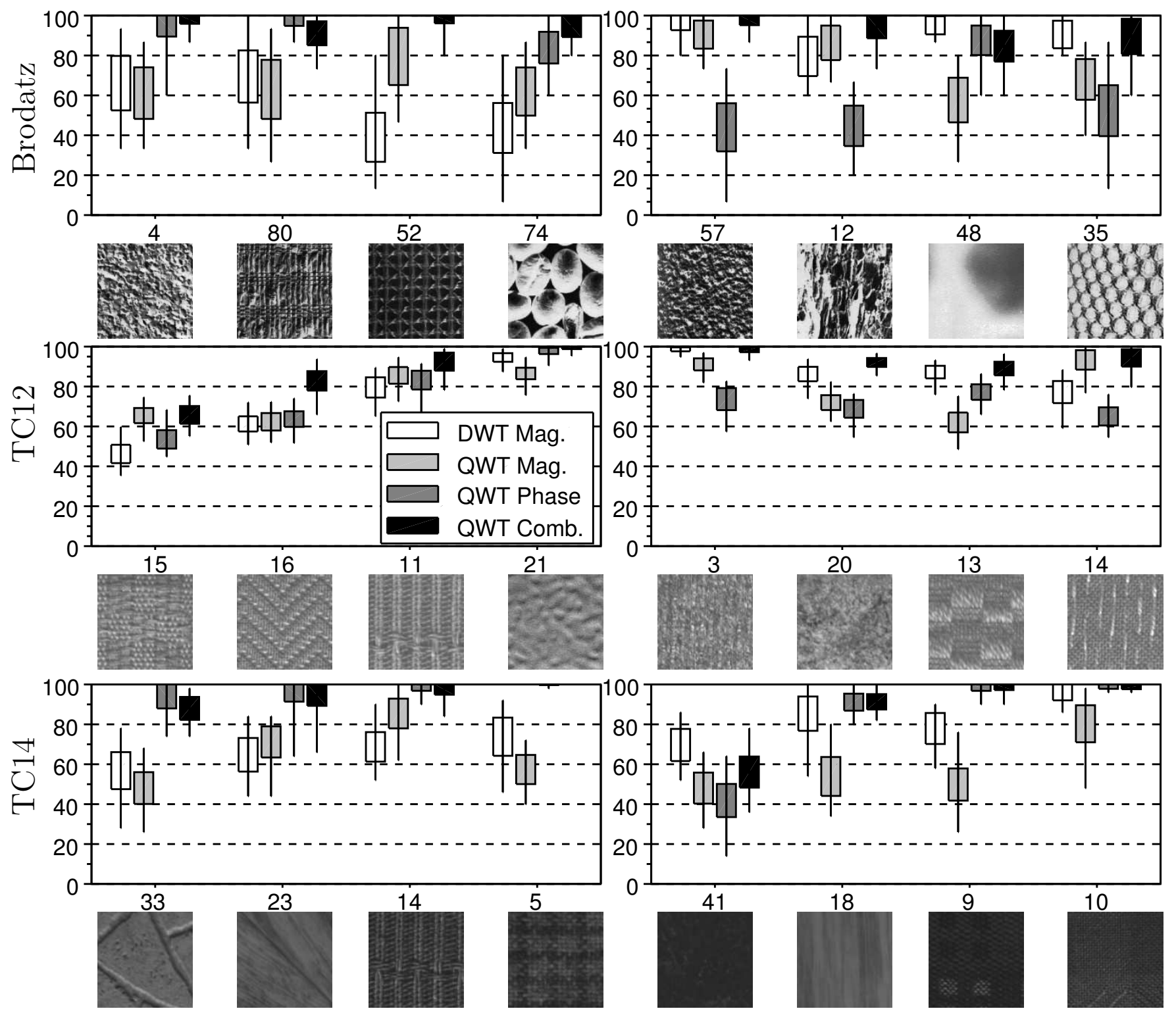

Figure 4: Cross validation results for some classes of the 3 databases. See also Fig. 3. Performance of each class is shown for the measures : DWT magnitude st. dev. (white), QWT magnitude st. dev. (light-gray), QWT phase weighted st. dev. (dark-gray) and QWT combination of magnitude and phase (black). 
Brodatz base :
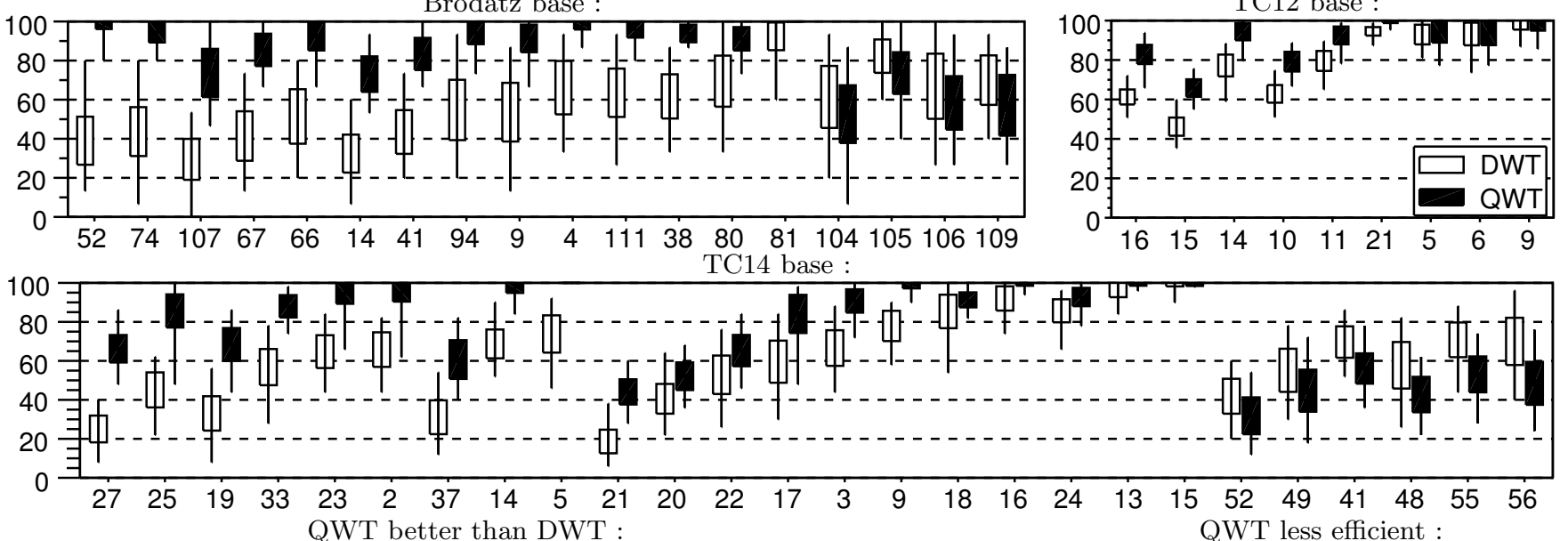

Brod. 52

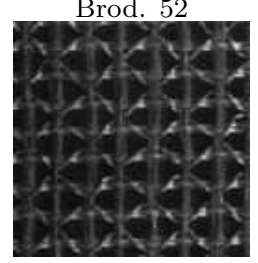

TC14 14

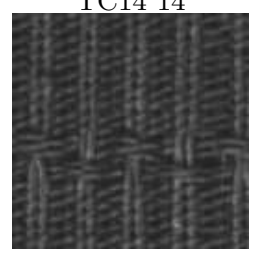

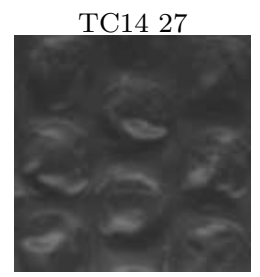

Brod. 66
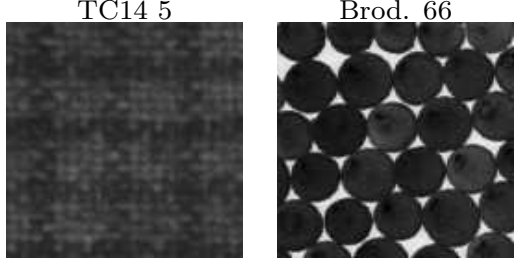

TC14 33

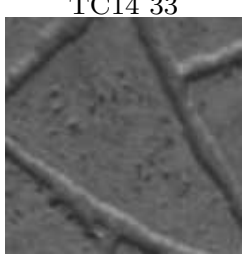

Brod. 67

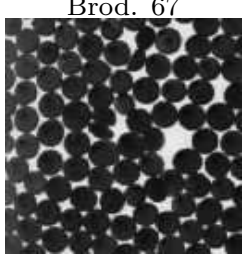

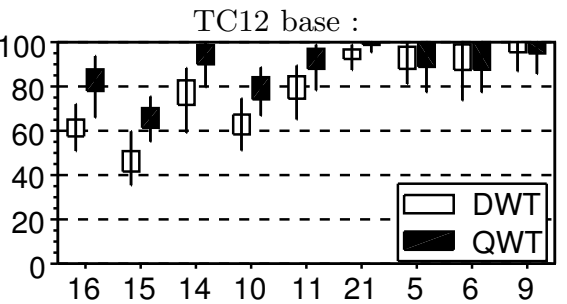

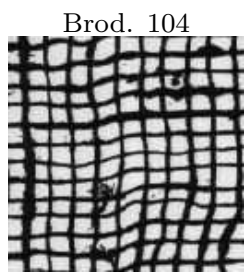

TC14 56

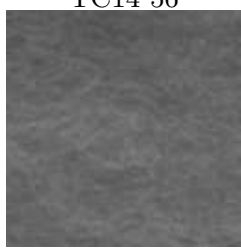

Brod. 105

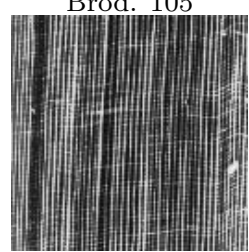

TC14 55

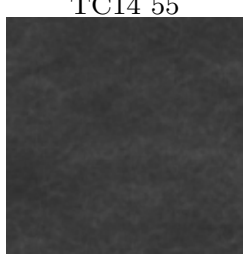

Figure 5: Final classifier performance for the DWT feature vector and the final QWT feature vector using both magnitude and phase.

provides additional geometric information.

We can hardly identify some kind of textures better recognized by either magnitude or phase but the recognition rates really show that magnitude and phase are completing one another; so it seems natural to combine both feature vectors. 


\begin{tabular}{|l|c|c|c|c|c|c|}
\hline & \multicolumn{2}{|c|}{ Brodatz } & \multicolumn{2}{c|}{ TC12 } & \multicolumn{2}{c|}{ TC14 } \\
\hline & Mean & Dev & Mean & Dev & Mean & Dev \\
\hline DWT Mag. & $64 \%$ & 9 & $83 \%$ & 4 & $56 \%$ & 8 \\
\hline QWT Mag. & $64 \%$ & 9 & $82 \%$ & 4 & $51 \%$ & 8 \\
\hline QWT Phase & $65 \%$ & 8 & $79 \%$ & 4 & $58 \%$ & 6 \\
\hline QWT Comb. & $\mathbf{7 6} \%$ & $\mathbf{7}$ & $\mathbf{9 1} \%$ & $\mathbf{4}$ & $\mathbf{6 3} \%$ & $\mathbf{6}$ \\
\hline CWT Mag. & $65 \%$ & 8 & $87 \%$ & 4 & $62 \%$ & 7 \\
\hline
\end{tabular}

Table 1: Final recognition rates.

\subsection{Combined QWT magnitude and phase}

The final feature vector based on QWT magnitude and phase provides very good classification performance with an improvement of about $10 \%$ recognition with respect to DWT. We present global recognition rates (mean rate and standard deviation of obtained rates according to cross validation) in table 1. As we cannot present every texture class we selected interesting cases - illustrated Fig. 4 - with recognition rates for respectively DWT magnitude based feature extraction, QWT magnitude, QWT phase, and final QWT feature vector. A sample is displayed under each class of the $x$-axis. We hope such an illustration will give some insight about visual texture characteristics that we could link with QWT properties. Fig. 5 shows more global results between DWT and QWT to conclude about the general improvement of classification thanks to the QWT. We have about a dozen 'bad' cases but the overwhelming majority of classes are better recognized by QWT and figure 5 is very convincing about its superiority.

Now we discuss some particular cases to analyze why the QWT is better 
or worse than DWT.

A first remark is about texture Brod. 52 that is strikingly better analyzed by the QWT phase feature vector with $100 \%$ recognition. The reason is this texture contains only horizontal, vertical and - mostly - diagonal structures that makes $\psi$ very significant.

Most of TC14 classes for which DWT is better are visually similar (see Fig. 5 TC14 55 and 56), almost uniform and hard to distinguish. These images have a pure stochastic behavior so the orthogonality of the DWT is better adapted than the structural analysis brought by QWT - yet this issue is open.

The same remark as in section 5.1 about shift-invariance still holds for textures Brod. 52, TC14 14 and TC14 5.

Note that most textures for which the single QWT magnitude is very worse than DWT (see Fig. 3) are better represented with the final feature vector which outperforms the DWT. This result shows that QWT magnitude and phase are strikingly complementary because both give separately bad results whereas very efficient when combined. Class TC12 20 (Fig. 4) makes a good example.

Finally, this use of quaternionic representation of textures greatly improves an analogous standard wavelet based texture analysis. We proposed a wavelet based feature vector that yields an average recognition rate about $10 \%$ better than standard wavelets; by using both QWT magnitude - containing similar information than DWT but with shift-invariance - and QWT phase that provides structural information. Standard DWT does not allow using such a phase - making it a less coherent representation. With this 
texture classification we validate the recent QWT from an application point of view as superior to standard DWT.

We will now present experimental results with $\mathbb{C W T}$. Recall that $\mathbb{C W T}$ coefficients are fundamentally different from QWT coefficients in terms of analysis and interpretation of their geometric meaning. Even if both are computed by a separable Dual-Tree algorithm the combination of the 4 related filterbank outputs is different. In the QWT case the 4 outputs are seen like Cartesian components of 3 quaternion valued subbands while for $\mathbb{C W T}$ some sums and differences of outputs are considered to be real and imaginary parts of 6 complex subbands - see [Selesnick et al., 2005].

This is why analysis provided by $\mathbb{C W T}$ has not much to do with QWT; and we will see in the next section that kinds of texture better recognized by QWT or $\mathbb{C W T}$ - as well as global performance - are not the same.

\subsection{Results with complex wavelets}

Results obtained with features from [Celik and Tjahjadi, 2009, 2011] have to be studied carefully. Recall that we present it to compare with literature and that we considered only the feature extraction part from cited references. So result we get are not comparable with the very high performance of original papers partly due to a clever choice of classification algorithm together with proper normalization and feature selection/normalization. In addition size of training bases may differ.

Globally, the first feature descriptor based on $\mathbb{C W T}$ magnitude provides better recognition than DWT but worse than final QWT based features; which is an expectable result. Second feature vector based on both $\mathbb{C W T}$ magnitude and phase gave very bad recognition (about 5\%). We observe that 

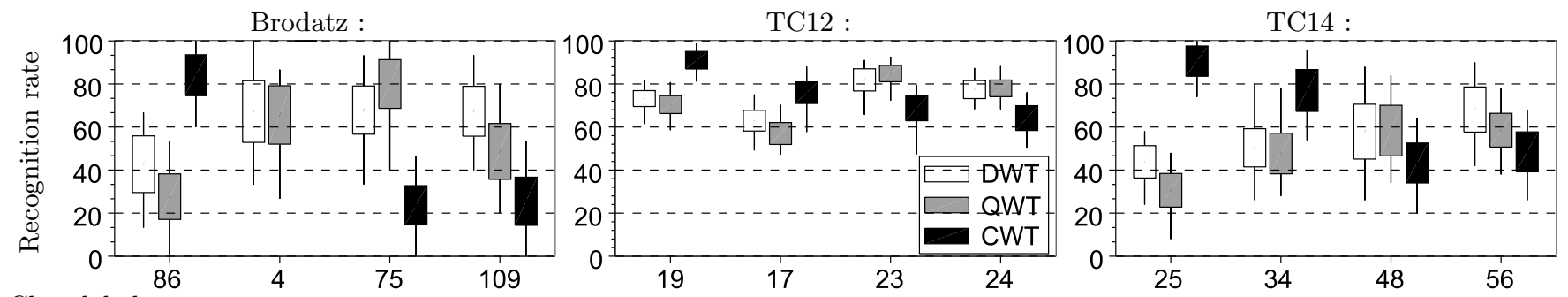

Class label

$\mathbb{C W T}$ more efficient :
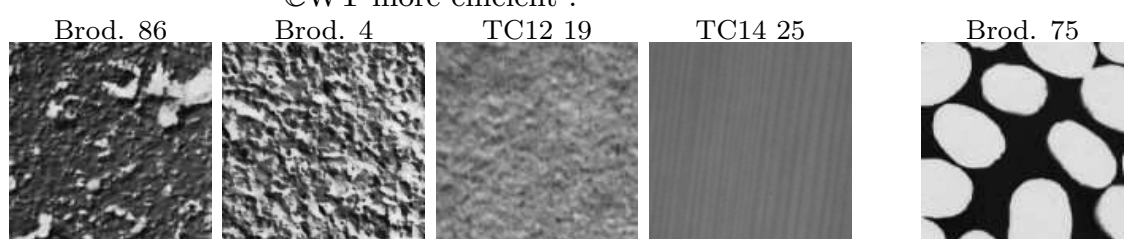

$\mathbb{C W T}$ less efficient :

$\begin{array}{lll}\text { TC12 } 23 & \text { TC12 } 24\end{array}$
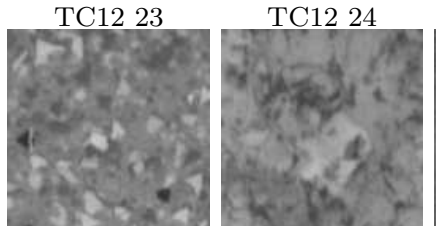

TC14 56

Figure 6: Complex wavelets based classification performance with feature vector described in [Celik and Tjahjadi, 2009]. We selected cases of biggest difference of performance between DWT/QWT and $\mathbb{C W T}$.

$\mathbb{C W T}$ phase measures are numerically unstable because no special weighting is used. In practice many absurd angles - related to the numerous zero coefficients - discredit variance end energy features processed on phase data. In [Celik and Tjahjadi, 2011] a PCA based feature selection is done before classification - what we did not do - so phase based features may be discarded by this stage. Thus we will only study magnitude based analysis from [Celik and Tjahjadi, 2009].

We show Fig. 6 classification results with the first feature vector [Celik and Tjahjadi, 2009] in comparison with DWT based st. dev. and QWT based final feature vector. Like previous figures, we present only cases of biggest difference between different methods.

As global rates lie between DWT and QWT (see table 1) we can conclude that - by restricting ourselves to simple global features - the oriented behavior 
of $\mathbb{C W T}$ together with shift-invariance of its magnitude can improve a DWT based analysis but the proposed QWT-phase analysis performs better.

An example of the advantage of $\mathbb{C W T}$ directionality is texture TC14 25 (Fig. 6). This texture class exhibits straight lines with an orientation around $75^{\circ}$. This is precisely the orientation on which one of the $6 \mathbb{C W T}$ subbands is aligned. The $\mathbb{C W T}$ is well suited in this very particular case so the recognition performance is much better than DWT/QWT.

However directional analysis brought by $\mathbb{C W T}$ is globally not as powerful as the 2D quaternionic phase concept of QWT. The 6 subbands of $\mathbb{C W T}$ offer better frequency selectivity than DWT but it still carry some similar "energy" information. On the other hand the fundamental relation between QWT $\psi$-phase and geometry provides a new kind of additional information that complements "energy" data. Finally our results completely confirm that quaternionic wavelets provide better geometric information than DWT and $\mathbb{C W T}$ for a texture analysis purpose.

\section{Conclusion}

The Quaternionic Wavelet Transform is a recent improvement of standard wavelets that aims to yield coefficients with a shift-invariant magnitude and a phase containing geometric information. This transform makes use of a 2D generalization of the analytic signal : a classical powerful tool for signal analysis. This generalization due to Bülow gives a coherent local analysis of 2D signals and was naturally embedded into a wavelet framework to overcome common drawbacks of standard wavelets. Redundancy brought by QWT phase adds complete structural information about local features of images. 
The QWT is not straightforward to interpret and requires a quite sophisticated theoretical framework. But here we gave an application study crossing the gap between that framework and the way to use this tool; by showing its superiority over standard wavelets in a texture analysis context. With a large texture database our QWT based feature extraction gives globally $10 \%$ better recognition than DWT. This improvement is due to shift-invariance of QWT magnitude together with the use of QWT phase that contains useful structural information for texture analysis.

A comparison is also made with complex wavelets that have been used in literature in similar works. Advantage of complex wavelets is good directionality; which is confirmed in practice by better recognition for some highly oriented textures. But its complex phase fails to provide such geometric information as this of QWT. As a result QWT outperforms complex wavelets.

Our classification algorithm is a first approach as we focus on studying QWT so there are many ways of improvements in terms of pattern recognition. In particular, spatial analysis of subbands is not used and the classification proposed in [Arivazhagan and Ganesan, 2003] for example could be improved by using a comprehensive use of QWT phase to provide an excellent classification that could compete with state of the art algorithms.

Our future work implies the monogenic wavelet transform - an improvement of the QWT based on another generalization of the analytic signal : the monogenic signal. This transform is harder to implement but can lead to a more coherent signal analysis thanks to better properties. Monogenic magnitude is rotation invariant and the phase is easier to interpret than QWT 
phase.

\section{Acknowledgements}

This work is supported by the ANR project VERSO - CAIMAN. We also would like to thank the anonymous reviewer for his/her valuable comments.

\section{References}

Arivazhagan, S., Ganesan, L., June 2003. Texture classification using wavelet transform. Elsevier Pat. Rec. Lett. 24 (9-10), 1513-1521.

Bayro-Corrochano, E., 2006. The theory and use of the quaternion wavelet transform. J. Math. Imaging Vis. 24 (1), 19-35.

Blanchet, G., Moisan, L., Rougé, B., 2008. Measuring the global phase coherence of an image.

URL http://hal .archives-ouvertes.fr/hal-00212337/en/

Brodatz, P., 1966. Textures: A Photographic Album for Artists and Designers. Dover publications, New York.

Bülow, T., 1999. Hypercomplex spectral signal representation for the processing and analysis of images. Thesis.

Celik, T., Tjahjadi, T., 2009. Multiscale texture classification using dual-tree complex wavelet transform. Pattern Recogn. Lett. 30 (3), 331-339.

Celik, T., Tjahjadi, T., 2011. Bayesian texture classification and retrieval based on multiscale feature vector. Pattern Recognition Letters 32 (2), $159-167$. 
Chan, W., Choi, H., Baraniuk, R., July 2008. Coherent multiscale image processing using dual-tree quaternion wavelets. IEEE Transactions on Image Processing 17 (7), 1069-1082.

de Rivaz, P., Kingsbury, N., 1999. Complex wavelet features for fast texture image retrieval. pp. I:109-113.

Hahn, S. L., 1996. Hilbert transforms in signal processing. Artech House, Boston, London.

Kingsbury, N., 2001. Complex wavelets for shift invariant analysis and filtering of signals. Applied and Computational Harmonic Analysis 10 (3), 234 $-253$.

Lasmar, N.-E., Berthoumieu, Y., 2010. Multivariate statistical modeling for texture analysis using wavelet transforms. Acoustics, Speech, and Signal Processing. Proceedings. (ICASSP). IEEE International Conference on.

Selesnick, I. W., Baraniuk, R. G., Kingsbury, N. G., 2005. The dual-tree complex wavelet transform. IEEE Signal Processing Magazine [123] November.

Tuceyran, M., Jain, A. K., 1993. Texture analysis. In: Handbook of pattern recognition and computer vision. World Scientific Publishing Co., Inc., River Edge, NJ, USA, pp. 235-276.

Vo, A., Oraintara, S., Nguyen, T., 2007. Using phase and magnitude information of the complex directional filter bank for texture image retrieval. pp. IV: 61-64. 
672 Zhou, J., Xu, Y., Yang, X., 2007. Quaternion wavelet phase based stereo 673 matching for uncalibrated images. Pattern Recogn. Lett. 28 (12), 1509$674 \quad 1522$. 